\title{
Metformin Causes Hepato-renal Dysfunctions in Obese Male Rats
}

\author{
Sabry Ali El-Naggar ${ }^{1^{*}}$ \\ https://orcid.org/0000-0002-3001-795X
}

Mona Elwan ${ }^{1}$

https://orcid.org/0000-0002-3214-4549

\section{Mohamed Abo Elftouh Basyouny ${ }^{1}$}

https://orcid.org/0000-0001-8073-5717

\section{Ehab Othman Elshennawy ${ }^{1}$ \\ https://orcid.org/0000-0002-8112-400X}

\section{Karim Samy El-Said ${ }^{2^{*}}$ \\ https://orcid.org/0000-0003-1511-6033}

${ }^{1}$ Tanta University, Faculty of Science, Zoology Department, Tanta, Egypt; ${ }^{2}$ Tanta University, Faculty of Science, Biochemistry Division, Chemistry Department, Tanta, Egypt.

Editor-in-Chief: Alexandre Rasi Aoki

Associate Editor: Sinvaldo Baglie

Received: 2021.03.26; Accepted: 2021.08.16.

*Correspondence: kareem.ali@science.tanta.edu.eg; Tel.: +201002977062 (K.S.E.)

\section{HIGHLIGHTS}

- Metformin reported to decrease body weight of obese patient.

- Metformin showed hepatorenal side effects in obese rats.

- Metformin induces biochemical changes in obese rats.

- Metformin alters histological architectures of liver and kidney of obese rats.

Abstract: Obesity results in detrimental effects on different body organs. Metformin (Met) has described to decrease the body weight of obese patient and to control the glucose level. This study aimed to evaluate the role of Met treatment for long period on the functionality of liver and kidney organs of obese rats. Forty rats were used in this study and divided into four groups as the following: group 1 (Gp1) was served as a negative control that administered orally with $200 \mu \mathrm{L}$ of $\mathrm{H}_{2} \mathrm{O}$. Gp2 of rats was administered with Met $(200 \mathrm{mg} / \mathrm{kg})$ daily for a month. Gp3 was obese rats, and Gp4 was obese rats administered with Met as in Gp2. All rats were sacrificed to analyze hematological, biochemical, and histopathological changes. The results showed that Met decreased the body weight of both naïve and obese rats, however, it caused hepato-renal dysfunctions in obese rats as evidenced by increased the levels of ALT, AST, urea, creatinine, and MDA and decrease in the antioxidants biomarkers (SOD, Cat and GSH). Collectively, Met causes liver and kidneys dysfunctions of obese rats and is not recommended to described for obese persons. 
Keywords: Metformin; Hepato-renal; Obesity; Antioxidants; Dysfunction.

\section{INTRODUCTION}

Obesity is typically defined as excess body weight. Obesity increases risk of chronic disease such as T2DM, cardiovascular disease and certain cancers [1]. Obesity causes insulin resistance, resulting in a series of obesity-related disorders [2]. Obese people have inadequate glycemic, blood pressure and other obesityrelated medical conditions [3]. Unfortunately, weight gain led to cardiovascular manifestations and other morbidity [4]. Weight reduction is an important part of therapy in all obese patients. For weight maintenance and loss, anti-diabetic drugs such as metformin can be potential in both weight and glucose control of obese diabetic patients [5].

Metformin (Met) is well-known as a promising hypoglycemic drug, particularly prescribed in patients with type 2 diabetes mellitus (T2DM) [6]. Met is useful in increasing insulin sensitivity, reducing gastrointestinal glucose absorption and reducing the incidence of the metabolic syndrome in obese patients [7,8]. Despite the known glucose-lowering effects of Met, clinical studies reported its promising role in weight loss via decreasing the appetite and regulation of fat oxidation and storage in liver and peripheral tissues [9]. The direct effect of Met on the signaling mechanisms to regulate food intake has been investigated [10]. It has been reported that Met induce mammalian target of rapamycin, a downstream target of adenosine monophosphate kinase (AMPK) that mediates the effects of leptin, suppresses the appetite, and decreases ectopic lipid stores [11,12]. Furthermore, it has been reported that Met could be used as anticancer drug especially for lung cancer [13]. Even though, the importance of Met on diabetic and cancer treatment has been addressed, till now a debate about the effect of Met on the renal function is standing. Siwei et al. (2017) reported that Met can improve kidney lesions and increase antioxidant activity of superoxide dismutase in diabetic rats [14]. Previous study showed an adverse effect of Met upon the treatment of diabetic persons [15]. There is strong and consistent evidence that obesity management can delay the progression from prediabetes to T2DM [16]. Some physicians described Met to obese persons to help them in losing weight. The present study designed to evaluate the impact of Met on the hepato-renal functions in high-fat diet (HFD)induced obese male rats.

\section{MATERIALS AND METHODS}

Metformin (Met) was purchased from local pharmacy in Tanta, Egypt, diluted by phosphate buffer saline (PBS) and the concentration was adjusted to $200 \mathrm{mg} / \mathrm{kg} \mathrm{b.wt} \mathrm{in} 200 \mu \mathrm{l}$ for oral administration. Cholesterol was purchased from Sigma-Aldrich (St Quentin Fallavier, France). All biochemical kits were purchased from Bio-diagnostic Company, Egypt.

Animals: Forty-two rats $(130 \pm 5 \mathrm{~g})$ allowed acclimating for 1 week in the animal house conditions of the Faculty of Science, Tanta University, before being divided into groups. Animals were used for the experimental design. The institutional animal care committee at Zoology Department, Faculty of Science, Tanta University-Egypt, approved the experimental design (IACUC-SCI-TU-00375). Target values for temperature and relative humidity were about $22 \pm 1^{\circ} \mathrm{C}$ and $55 \pm 5 \%$ respectively, light- dark (day/night) cycle was achieved. Rats were given drinking tap water and normal experimental pelleted animal food ad libitium. Animals were carefully observed every day and their body weights, while food consumption and water intakes were measured precisely every week to evaluate any signs of toxicity or abnormality during the experiment.

Obesity induction and experimental design: Rats were divided into four groups ( $n=10)$. Group 1 (Gp1) and group 2 (Gp2) were given drinking tap water and normal experimental pelleted animal food ad libitium, while group 3 (Gp3) and group 4 (Gp4) were given drinking tap water and high-fat diets (HFD) contentiously for 5 weeks to induce obese rats. Then, Gp1 administered orally with saline, while Gp2 received Met as 200 $\mathrm{mg} / \mathrm{kg}$, once daily by oral gavage for 30 days. Group 3 (Gp3) received saline and Gp4 received Met as 200 $\mathrm{mg} / \mathrm{kg}$, once daily by oral gavage as in Gp2. At the end of the experiment, all rats were sacrificed under ethyl ether anesthesia and gross examinations were performed macroscopically on all groups during sacrifice.

Determination the percentage of the change on total body weight: All groups of rats were weighted at the beginning (initial b.wt) and at the end of the experiment (final b.wt). The percentage of the change in the total body weight (\% T.B.W) was calculated as follow: (final b.wt - initial b.wt / initial b.wt) $\times 100$.

Hematological and biochemical analysis: To determine the hematological parameters, the blood was withdrawn to estimate the total count of red blood cells (R.B.Cs), hemoglobin content (Hb), hematocrit (Hct\%), the total count of white blood cells (W.B.Cs) and differential count using auto hematology analyzer (BC-3200, Mindray, China). The values were then compared with the normal and standard values. Serum alanine 
aminotransferase (ALT) and aspartate aminotransferase (AST) were determined as described [17]. Serum cholesterol, triglycerides, and high-density lipoprotein cholesterol (HDL) were determined using quantitative kit based on the previously described methods [18-20]. Low-density lipoprotein cholesterol (LDL) was calculated as follows: $\mathrm{LDL}=$ total cholesterol- HDL- VLDL [21]. Malondialdehyde (MDA), superoxide dismutase (SOD), catalase (CAT), and reduced glutathione (GSH) were determined according to their manufacturing protocols [22-24].

Histopathological investigations: Tissue specimens of the liver were harvested and fixed in $10 \%$ formalin. Paraffin blocks were prepared after completing the tissue processing in different grades of alcohol and xylene. Sections $(5 \mu \mathrm{m})$ were prepared from paraffin blocks using microtome, stained with hematoxylin and eosin, and observed under a light microscope (Optica light microscope, B- 350) to examine gross cellular damage.

Statistical analysis: One-way analysis of variance (ANOVA) was used to assess the significant differences among treatment groups. Dunnet test was used to compare all groups against the control group to show the significant effect of treatment. The criterion for statistical significance was set at $p<0.05$. All data are presented as mean \pm SEM.

\section{RESULTS}

Rats were fed with high fat diet (HFD) for five weeks to induce obesity in rats. Post 5 weeks of feeding on HFD, the body weight of rats increased from 150 to $250 \mathrm{~g}$ with percentage of change (66.6\%) (Figure 1 ). Then, naïve, and obese rats were administrated either with dist. $\mathrm{H}_{2} \mathrm{O}$ or with Met orally for 30 days every day. As compared to the control group (Gp1), naive rats/Met group (Gp2) showed low percentage of body weight changes. Obese rats/Met group (Gp4) showed low percentage of body weight changes when compared to group of obese rats (Gp3).
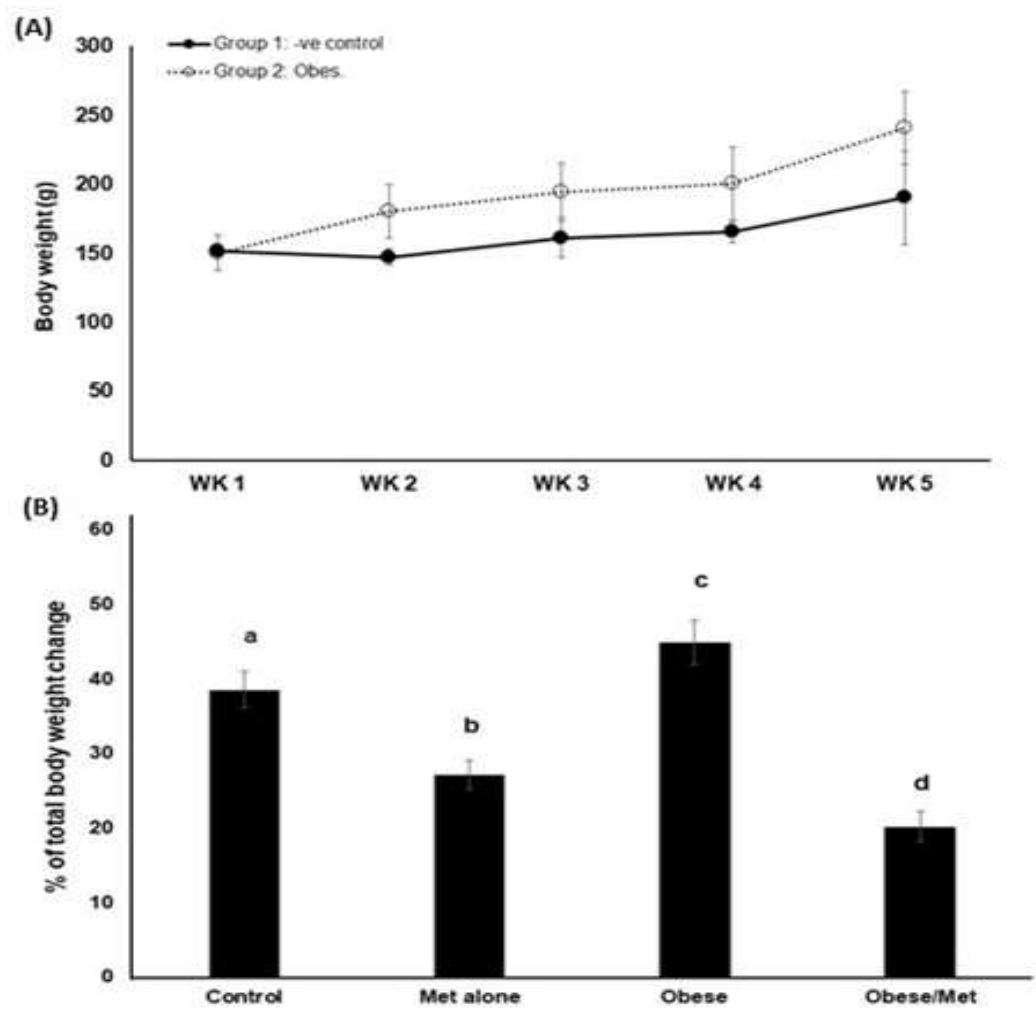

Figure 1 ( $A$ and $B)$. Kinetic changes of total body weight of control rats fed on normal diet and obese-induced rats fed on HFD (A). Percentages of total body weight changes of both naïve and obese rats post treatment with Met for two months $(B)$.

The results showed that the hemoglobin $(\mathrm{Hb})$ level, total red blood cell (RBCs) counts, hematocrit (Hct) \%, total white blood cells (WBCs) and platelets count did not significantly change among all groups under the study (Table 1). 
Table 1. Hematological parameters in different groups of rats treated with Met.

\begin{tabular}{llllll}
\hline Groups & $\mathbf{H b}(\mathbf{g} / \mathbf{d L})$ & RBCs $\left(\mathbf{x 1 0 ^ { 6 } / \mu L )}\right.$ & Hct $(\%)$ & WBCs $\left(\mathbf{x 1 0 ^ { 3 } / \mu L )}\right.$ & Plat. $\left(\mathbf{x 1 0 ^ { 3 } / \mu L )}\right.$ \\
\hline Naïve & $11.82 \pm 0.37$ & $8.82 \pm 0.41$ & $39.3 \pm 3.3$ & $13 \pm 0.5$ & $882.8 \pm 31.3$ \\
Naïve/Met & $11.0 \pm 0.5$ & $8.71 \pm 0.1$ & $41.9 \pm 2.5$ & $12 \pm 0.8$ & $740 \pm 78.2$ \\
Obese & $14.53 \pm 0.46$ & $8.81 \pm 0.22$ & $44.7 \pm 2.9$ & $11 \pm 0.25$ & $699 \pm 68.5$ \\
Obese/Met & $14.0 \pm 0.4$ & $8.5 \pm 0.27$ & $45 \pm 3.8$ & $10 \pm 0.6$ & $778 \pm 31.1$ \\
\hline
\end{tabular}

The values represented mean \pm SD. $p$ value $<0.05$ was statistically significant. Means that do not share a letter are significantly different.

The levels of alanine transaminase (ALT) and aspartate transaminase (AST) in naive/Met group (Gp2) did not change when compared to control group (Gp1). However, the levels of ALT and AST were significantly increased in obese group when compared to the naive rats (Gp1). Obese/Met group (Gp4) showed an increase in the level of AST and ALT when compared to obese rats (Gp3). The levels of urea and creatinine did not show significant changes in naïve/Met group (Gp2) when compared to Gp1 (Table 2). In obese rats, the level of urea increased; however, the level of creatinine did not change when compared to Gp1. In obese/Met group (Gp4), the levels of urea and creatinine were increased when compared to obese rats (Gp3). Treatment of obese/Met group showed the highest level of urea and creatinine among all groups (Table 2).

Table 2. ALT, AST, urea, and creatinine levels in the sera of different experimental groups.

\begin{tabular}{llllc}
\hline Group & GPT (U/L) & GOT (U/L) & Urea $(\mathbf{m g} / \mathbf{d L})$ & Creatinine $(\mathbf{m g} / \mathbf{d L})$ \\
\hline Naive & $69.3 \pm 4.16^{\mathrm{c}}$ & $130.0 \pm 2.65^{\mathrm{c}, \mathrm{d}}$ & $22.00 \pm 2.0^{\mathrm{e}}$ & $0.62 \pm 0.09$ \\
Naïve/Met & $70.3 \pm 8.39^{\mathrm{c}}$ & $121.0 \pm 1.00^{\mathrm{d}}$ & $25.00 \pm 2.0^{\mathrm{d}, \mathrm{e}}$ & $0.72 \pm 0.03$ \\
Obese & $78.3 \pm 5.03^{\mathrm{b}, \mathrm{c}}$ & $155.0 \pm 5.00^{\mathrm{b}}$ & $34.67 \pm 4.5^{\mathrm{b}, \mathrm{c}}$ & $0.63 \pm 0.07$ \\
Obese/Met & $95.0 \pm 4.0^{\mathrm{a}}$ & $126.7 \pm 5.86^{\mathrm{c}, \mathrm{d}}$ & $52.67 \pm 2.52^{\mathrm{a}}$ & $0.79 \pm 0.08$ \\
\hline
\end{tabular}

The values represented mean \pm SD. $p$ value $<0.05$ was statistically significant. Means that do not share a letter are significantly different.

Cholesterol, triglycerides, LDL, HDL and VLDL levels in naïve/Met group did not change significantly when compared to control group (Gp1). However, cholesterol, triglycerides, LDL and VLDL levels were increased in the obese rats (Gp3) when compared to Gp1. In contrast, the level of HDL in obese rats (Gp3) showed substantial decrease when compared to Gp1. In obese/Met group, the level of cholesterol was decreased when compared to obese rats. The level of HDL was decreased in obese rats (Gp3) when compared to Gp1. However, the level of LDL increased significantly in Gp3. In contrary, administration of Met in obese rats decreased LDL level (Table 3).

Table 3. Cholesterol, TG, HDL, LDL and VLDL levels in the sera of different experimental groups.

\begin{tabular}{|c|c|c|c|c|c|}
\hline Group & Cholesterol & TG & HDL & LDL & VLDL \\
\hline Naïve & $43.3 \pm 1.5^{c}$ & $53.00 \pm 2.65^{c}$ & $38.3 \pm 1.15^{a, b}$ & $10.00 \pm 1.0^{b}$ & $14.00 \pm 1.0^{c}$ \\
\hline Naive/Met & $41.0 \pm 1.0^{c}$ & $51.00 \pm 1.00^{c}$ & $36.3 \pm 1.5^{b}$ & $7.00 \pm 1.0^{\mathrm{b}}$ & $11.00 \pm 1.0^{d}$ \\
\hline Obese & $76.0 \pm 4.0^{\mathrm{a}}$ & $142.67 \pm 5.5^{a}$ & $42.0 \pm 3.6^{a, b}$ & $3.00 \pm 1.0^{c}$ & $28.33 \pm 1.5^{a}$ \\
\hline Obese/Met & 54. $3 \pm 2.5^{b}$ & $87.33 \pm 2.08^{b}$ & $38.3 \pm 2.1^{a, b}$ & $2.33 \pm 1.15^{c}$ & $17.00 \pm 1.0^{\mathrm{b}}$ \\
\hline
\end{tabular}

The values represented mean \pm SD. $p$ value $<0.05$ was statistically significant. Means that do not share a letter are significantly different.

The levels of superoxide dismutase (SOD), catalase (CAT), reduced glutathione (GSH) and malondialdehyde (MDA) in liver tissues of naïve/Met group did not change when compared to Gp1. The levels of SOD, CAT and GSH in liver tissues were decreased in both of obese rats and obese/Met group when compared to Gp1 and Gp2 (Figure 2). The levels of SOD, CAT and GSH in obese/Met group was higher than their levels in obese rats (Gp3). The level of MDA was increased in both of obese and obese/Met rats when compared to control groups. Interestingly, the level of MDA in obese/Met group was less than its level in obese rats (Figure 2). 
(A)

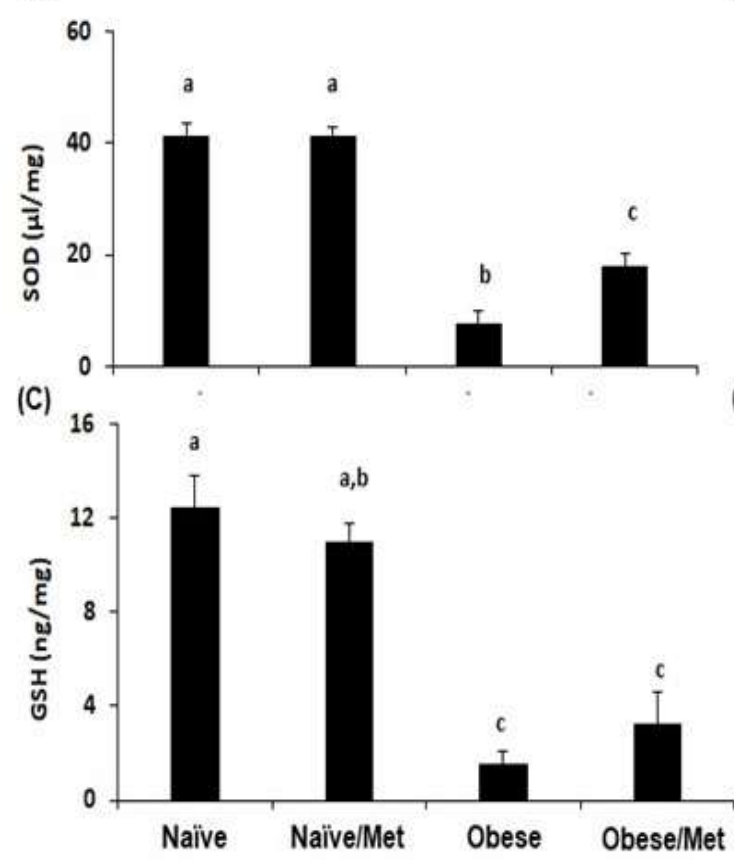

(B)

(D)
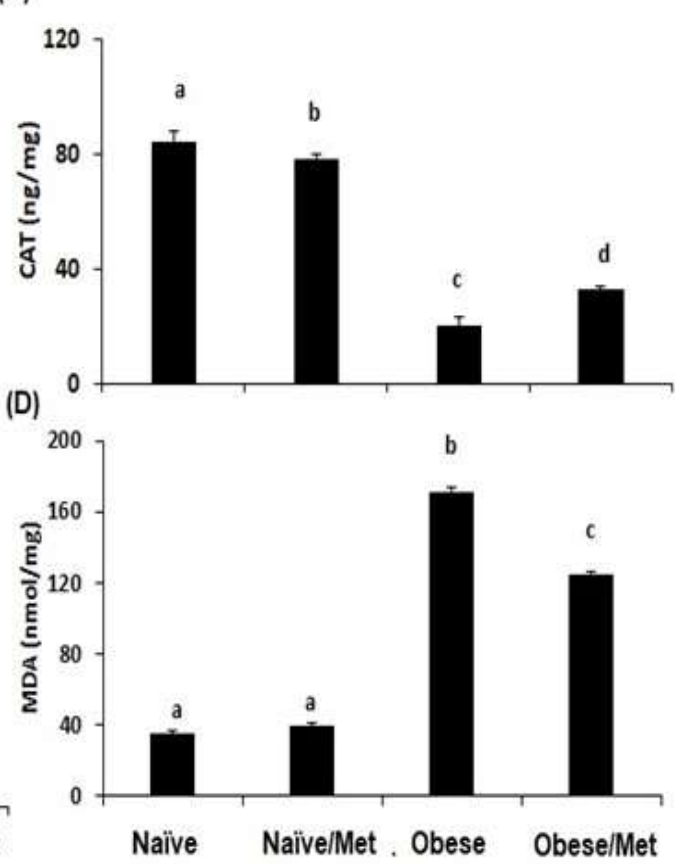

Figure 2. Malondialdehyde (MDA), superoxide dismutase (SOD), catalase (CAT), reduced glutathione (GSH) levels in the liver tissues of the different experimental groups.

In kidney tissues, the levels of SOD, CAT, GSH and MDA did not alter significantly in naïve/Met when compared to Gp1. The levels of SOD, CAT and GSH in kidneys tissues were decreased in both of obese and obese/Met groups when compared to control group. The levels of SOD, CAT and GSH in obese/Met rats was higher than their levels in obese rats. The level of MDA was increased in both of obese and obese/Met groups, even though, the level of MDA in obese/Met group was less than its level in obese rats (Gp3) (Table 4).

Table 4. MDA, SOD, CAT, GSH levels in the kidney tissues of the different experimental groups.

\begin{tabular}{llccc} 
Group & MDA $(\mathbf{n m o l} / \mathbf{m g})$ & SOD $(\boldsymbol{\mu L} / \mathbf{m g})$ & CAT $(\mathbf{n g} / \mathbf{m g})$ & GSH $(\mathbf{n g} / \mathbf{m g})$ \\
\hline Naïve & $34.00 \pm 3.16^{\mathrm{e}}$ & $38.5 \pm 1.29^{\mathrm{a}}$ & $76.25 \pm 2.22^{\mathrm{a}}$ & $12.0 \pm 0.82^{\mathrm{a}}$ \\
Naïve/Met & $36.25 \pm 3.86^{\mathrm{e}}$ & $33.5 \pm 1.29^{\mathrm{b}}$ & $72.25 \pm 1.71^{\mathrm{a}}$ & $11.75 \pm 0.96^{\mathrm{a}}$ \\
Obese & $120.50 \pm 1.29^{\mathrm{b}}$ & $19.75 \pm 1.7^{\mathrm{d}}$ & $38.00 \pm 2.16^{\mathrm{d}}$ & $5.94 \pm 0.43^{\mathrm{b}}$ \\
Obese $/$ Met & $148.25 \pm 3.30^{\mathrm{a}}$ & $12.0 \pm 2.94^{\mathrm{e}}$ & $21.50 \pm 1.29^{\mathrm{e}}$ & $1.74 \pm 0.21^{\mathrm{c}}$ \\
\hline
\end{tabular}

The values represented mean \pm SD. $p$ value $<0.05$ was statistically significant. Means that do not share a letter are significantly different.

Stained sections of the liver of control group (Gp1) showed a normal architecture with hepatic lobulation. The hepatic strands are alternating with narrow blood sinusoids that are lined by a layer of endothelial cells having the Kupffer cells (Figure 3A). Liver sections of naïve/Met group exhibits normal radiating hepatocytes with granular stained cytoplasm and centrically organized nuclei, few numbers of hepatocytes were degenerated, the central vein (Cv) is irregular and engorged with few RBCs, normal regular dilated blood sinusoids in between hepatic cords with normal Kupffer cells (Figure 3B). Liver sections of obese rats (Gp3) revealed notable degree of restoration of it's normal like structures, represented by regular arrangement of hepatocytes around $\mathrm{Cv}$, their cytoplasm acquires its homogenous stain and granular cytoplasm, but some hepatocytes with no obvious boundaries, some nuclei appear densely stained (pyknotic), others are faint stained ones (karyolitic) although some of them were still unchanged, irregular $\mathrm{Cv}$, and dilatation blood sinusoids (Figure 3C). Histopathological alterations were observed in Gp4 include noticeable disorganization of hepatic architecture, and congested Cv, mostly hepatocytes show degenerative and vacuolated cytoplasm with demarcated boundaries, pronounced nuclear changes like pyknotic nuclei and karyolitic ones, and deteriorative blood sinusoids were noticed (Figure 3D). 
Examination of renal cortex of the kidney sections of control rats (Gp1) showed normal appearance. The glomerulus is enclosed by two layers of epithelium, surrounded by Bowman's capsule. The glomeruli are round and oval, the proximal convoluted tubules were lined with simple epithelia. These epithelia had an acidophilic cytoplasm, and the apex possesses abundant microvilli which formed a brush border. The distal convoluted tubules were lined with simple cuboidal epithelium (Figure 4A). The kidney sections from rats that treated with Met (Gp2) exhibited renal profile is nearly like its normal structure. Most of the glomeruli were regular surrounded with prominent Bowman's capsule, some histopathological changes were noticed such as few numbers of the renal tubules were damaged and destructed, some nuclei of their lining epithelium are dark stained (pyknotic nuclei) as well as other renal tubules occluded with hyaline casts compared to the normal histological structure (Figure 4B). Investigated kidney sections of obesity rats (Gp3) showed normal like structure of the glomeruli that appear oval or rounded shape with normal appearance of the mesangial area, mostly of the renal tubules are normal, others with atrophy and destruction of its lining epithelium as well as their contents intermixed with each other (Figure 4C). The investigation of the kidney sections of obese/Met group (Gp4) revealed atrophy and disorganization of the glomeruli and irregular mesangial areas, few numbers of the renal tubules are normal but the mostly ones are disorganized, lost their characteristic appearance and their contents are intermixed with each other as well as intratubular hemorrhage was noticed (Figure 4D).

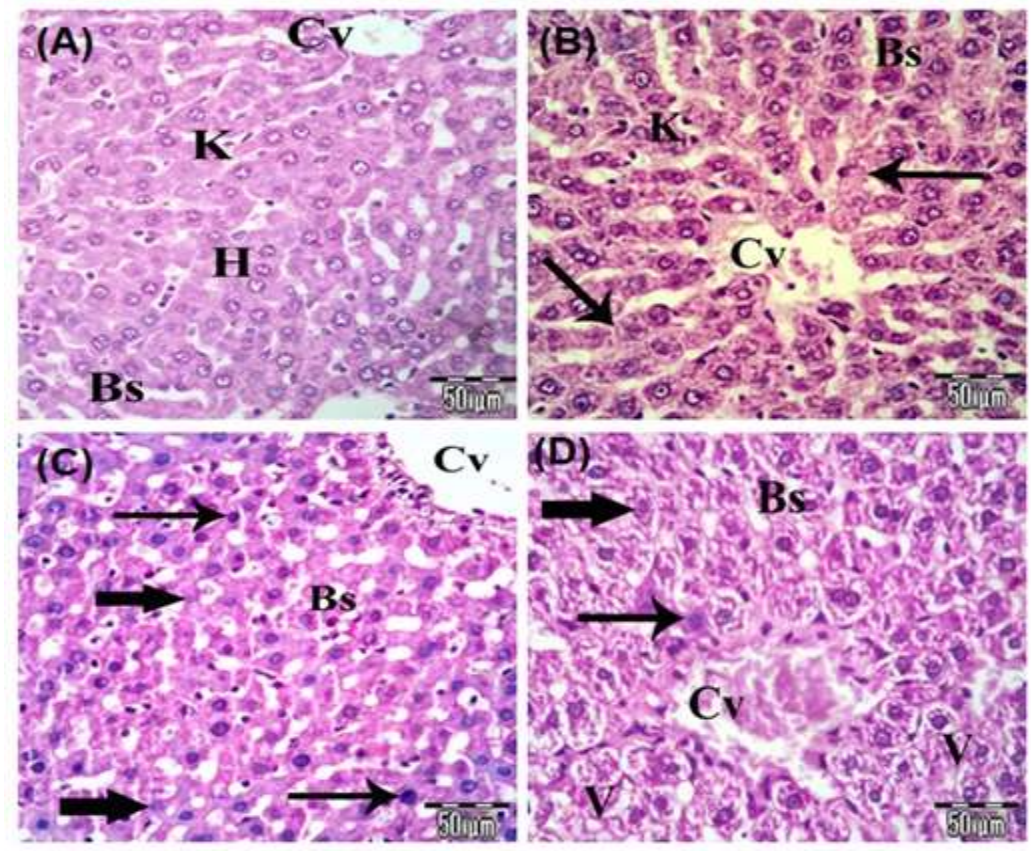

Figure 3. Liver sections of different experimental groups stained with H\&E. (A) High magnified section of control liver (Gp1) showing the normal histological structure of hepatic architecture, central vein (Cv) and radiating polygonal hepatocytes $(\mathrm{H})$. The hepatocytes had homogenous granular cytoplasm. The liver strands were alternating with narrow blood sinusoids (Bs) and distinct phagocytic Kupffer cells (K). (B) Liver section of naïve/Met group (Gp2) exhibits normal radiating hepatocytes $(\mathrm{H})$ with granular stained cytoplasm and centrically organized nuclei, few numbers of hepatocytes were degenerated (arrows), irregular Cv and normal regular dilated Bs. (C) Liver section of obese rats (Gp3) revealed some intact like hepatocytes, granular cytoplasm with no obvious boundaries and centric nuclei, some nuclei appear densely stained (pyknotic) (arrows), others are faint stained ones (karolitic) (thick arrows), irregular Cv, irregular dilated Bs. (D) Liver section of obese/Met group (Gp4) showing noticeable disorganization of hepatic architecture, congested $\mathrm{Cv}$, degenerative hepatocytes and vacuolated cytoplasm (V) with demarcated boundaries, non-organized nuclei. Some dark stained nuclei (pyknotic) (arrow), others are faint stained (karyolitic) (thick arrows) with deteriorative Bs. 

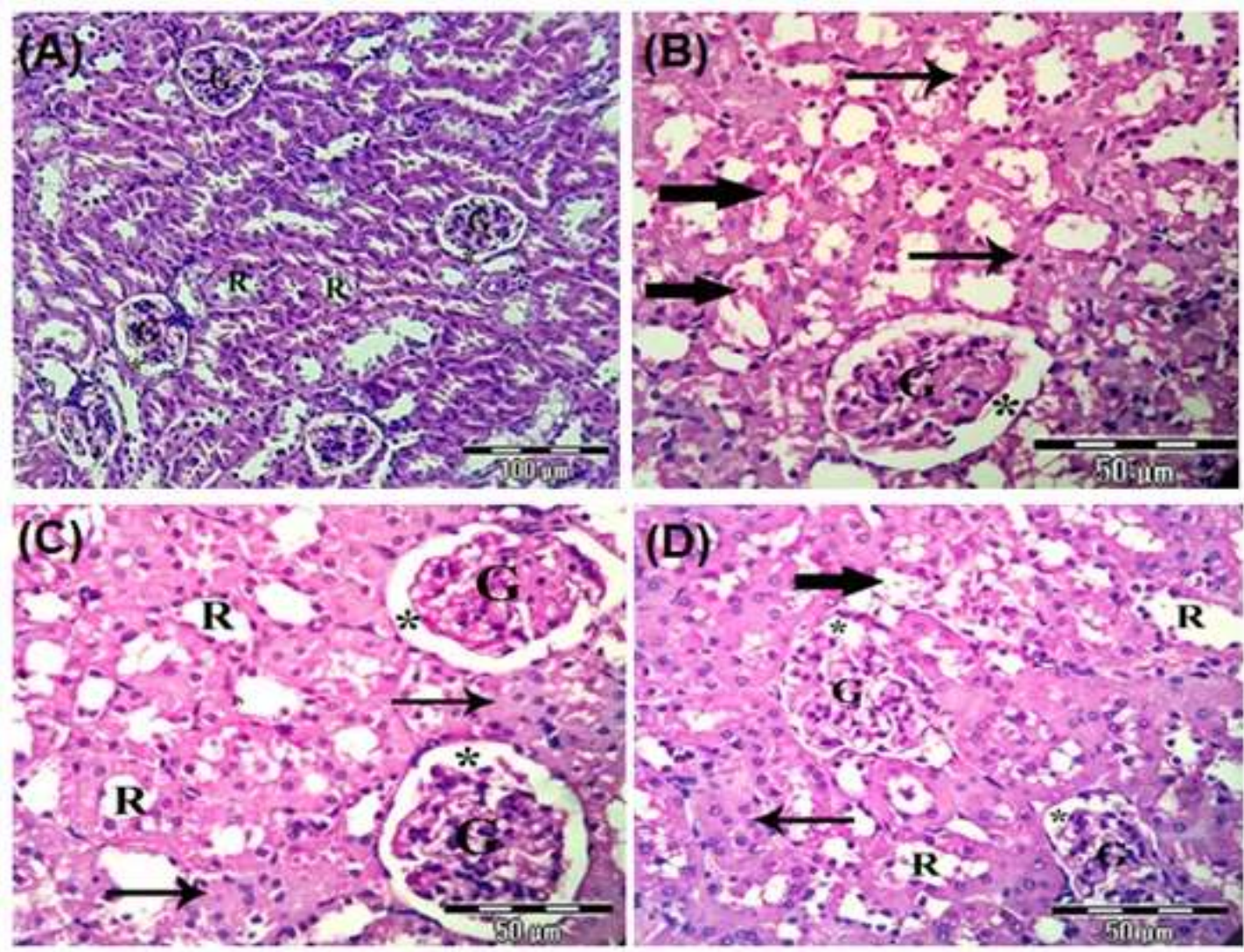

Figure 4. Kidney sections of different experimental groups stained with H\&E (X 400). (A) High magnified section of control rats (Gp1) showing normal architecture of renal glomeruli $(G)$ and renal tubules (R). (B) Kidney section of Gp2 (naïve/Met) exhibits normal like structure of the glomeruli $(G)$ and mesangial areas ${ }^{*}$ ), few numbers of the renal tubules were damaged and destructed, some nuclei of their lining epithelium are dark stained (pyknotic nuclei) (arrows) and renal tubules occluded with hyaline casts (thick arrows). (C) Kidney section of obese rats showing normal rounded or oval shape of the glomeruli $(G)$ and regular mesangial areas $\left({ }^{*}\right)$, mostly of the renal tubules $(R)$ are normal, others are damaged with atrophy and destruction of its lining epithelium. (D) Kidney section of obese/Met group (Gp4) reveals atrophy and disorganization of the glomeruli and irregular mesangial areas ${ }^{*}$ ), few renal tubules are normal but the mostly ones are disorganized, lost their characteristic appearance and their contents are intermixed with each other (arrows) as well as intratubular hemorrhage was noticed (thick arrow).

\section{DISCUSSION}

Obesity is a public health problem associated to T2DM, resulted from disordered in energy balance that increasing body fat mass. The anti-T2DM drug, Met which increase insulin sensitivity and help in losing weight [7]. Additionally, Met is found to have anti-inflammatory and antioxidant properties by increasing the production of mitochondrial reactive oxygen species (ROS) [25]. Met is generally considered to be safe in clinical practice, with gastrointestinal side effects such as nausea, dyspepsia, abdominal bloating, abdominal cramps, and diarrhea are commonly associated with it [26]. This study showed that feeding with HFD-induced obesity in male rats by increasing the total body weights when compared to rats fed on normal diet. This finding agreed with other study showed that HFD induce obesity in rats [27]. In this study, treatment of obese rats with Met led to weight loss and this may be due to the anorectic effect of this drug [9]. Treatment of naïve or obese rats with Met did not show significant changes in the examined hematological parameters, however, the platelets count slightly decreased in obese rats. Previous study showed that obesity could lead to an increase in the total number of white blood cells (WBCs). However, Met treatment cause decrease the total number of WBCs [28]. In the present study, treatment of naïve rats with Met did not alter the levels of AST and ALT in liver tissues, and this could explain that there is no toxicity post administration with Met. This finding agreed with a previous study reported that Met administration is safe for long period in normal rats [29]. The level of AST and ALT in obese rats, however, were increased when compared to naïve rats. Treatment of obese rats with Met additionally increased the levels of AST and ALT which could be indicates that Met treatment led to some toxicity on the liver tissues of obese rats. Previous study reported that Met treatment led to a hepatotoxicity, which in turn lead to an increase in the levels of AST, ALT. In contrast to our finding, Met treatment showed significant reduction in liver enzymes ALT and AST; this finding was 
reported by other researchers [30,31]. Treatment of control rats with Met for prolonged time did not alter the level of urea, and creatinine. This finding was consistent with previous study that reported the safety of Met on normal [32]. In obese rats, however, the level of urea and creatinine significantly increase than control rats. This finding agreed with previous report showed obesity increase the levels of urea and creatinine [33]. The results showed also that treatment of obese rats with Met increase the level of urea and creatinine than their levels in obese rats only. Again, this finding could illustrate the toxic effect of Met treatment on the kidney function of obese rats. Prolonged treatment of naïve rats with Met led to no changes in the lipid profile (cholesterol, triglycerides, LDL, HDL and VLDL). Obese rats showed significant increase in the previous lipid profile except the level of HDL was decreased. In contrast, treatment of obese rats with Met led to significant decrease in the levels of cholesterol, triglycerides, LDL and VLDL and increase in HDL levels. This could be explaining the beneficial effect of Met to ameliorate the lipids disorders induced by obesity. Several studies showed the direct effect of Met on the lipid profile [34,35].

The results showed that treatment of naïve rats with Met for prolonged time did not induce significant changes in the levels of SOD, CAT, GSH and MDA. This finding clearly showed no side effect of Met on the antioxidant status in normal rats [36]. In obese rats, however, the level of these antioxidant/oxidants' biomarkers changed, the level of SOD, CAT, and GSH were decreased, however, the level of MDA was increased. Treatment of obese rats with Met for prolonged time decreased the antioxidants biomarkers (SOD, CAT and GSH) and increased the levels of oxidative stress biomarker (MDA), when compared to their levels in obese rats. This finding indicated that the treatment with Met did not show improvement in the antioxidant capacity of obese rats.

Histopathological observations revealed noticeable alterations in the liver tissues of Met/obese group include marked disorganization of hepatic architecture, dilation and congestion of blood vessels, degeneration and vacuolation of mostly hepatocytes, pronounced nuclear changes like pyknotic nuclei and karyolitic ones. Our results contrast with previous study reported that treated obese rats with metformin can correct obesity in addition to improve liver function and histological response [36]. Also, our study showed that there are obvious histological alterations in the kidney tissues of Met/obese group that exhibits atrophy and disorganization of the glomeruli, disorganization of the mostly renal tubules as well as intratubular hemorrhage was noticed. While the kidney tissues of both Met and obese groups exhibited renal profile is nearly similar to its normal structure. Most of the glomeruli were normal and regular, some histopathological changes were noticed such as few numbers of the renal tubules were damaged, destructed, and occluded with hyaline casts.

\section{CONCLUSION}

Collectively, the data of the present study showed that obese rats administered with Met, an increase in liver, kidney functions biomarkers, and lipids peroxidation were observed when compared with normal control. Furthermore, the antioxidants biomarkers were decreased accompanied with alterations in histological architectures of liver and kidney tissues. Therefore, the prolonged treatment with Met revealed a hepatorenal dysfunctions in high fat diet-induced obese male rats.

Conflict of interest: Authors declare that there was no conflict of interest.

\section{REFERENCES}

1. Afshin A, Forouzanfar MH, Reitsma MB, Sur P, Estep K, Lee A, et al. Health effects of overweight and obesity in 195 countries over 25 years. New England J of Med. 2017; 377(1):13-27.

2. Barth RJ. Insulin resistance, obesity, and the metabolic syndrome. South Dakota J of Med. 2011; 22:1-7.

3. Rothberg AE, McEwen LN, Kraftson AT. Impact of weight loss on waist circumference and the components of the metabolic syndrome. BMJ Open Diabetes Researrch Care. 2017; 5:e000341.

4. Anderson JW, Kendall CW, Jenkins DJ. Importance of weight management in type II diabetes: review with metaanalysis of clinical studies. Americ College of Nut. 2003; 22:331-339.

5. Hollander P. Anti-diabetes and anti-obesity medications: effects on weight in people with diabetes. Diabetes Spectrum. 2007; 20(3):159-165.

6. Nasri H, Rafieian-Kopaei M. Metformin: Current knowledge. Int J of Res in Med Sci. 2014; 19(7):658-664.

7. Desilets AR, Dhakal-Karki S, Dunican KC. Role of metformin for weight management in patients without type 2 diabetes. Ann. Pharmacother. 2008; 42:817-26. 
8. Viollet B, Guigas B, Sanz Garcia N, Leclerc J, Foretz M, Andreelli F. Cellular and molecular mechanisms of metformin: an overview. Clin Sci. 2012; 122:253-70.

9. Malin SK, Kashyap SR. Effects of metformin on weight loss: potential mechanisms. Current opinion in endocrinology. Diab. Obes. 2014; 21(5):323-9.

10. Lv WS, Wen JP, Li L, Sun RX, Wang J, Xian YX, et al. The effect of metformin on food intake and its potential role in hypothalamic regulation in obese diabetic rats. Brain Res. 2012;1444:11-9.

11. Lim C, Kola B, Korbonits M. AMPK as a mediator of hormonal signaling. J. Molec. Endocrinol. 2010; 44:87-97.

12. Stevanovic D, Janjetovic K, Misirkic M, Vucicevic L, Sumarac-Dumanovic M, Micic D, et al. Intracerebroventricular administration of metformin inhibits ghrelin-induced hypothalamic AMP-kinase signaling and food intake. J. Neuroendocrinol. 2012; 96:24-31.

13. Xun C, Zhe-Sheng W, Xu-Dong W, Li Y, Liu K, Wang X. The clinical effect of metformin on the survival of lung cancer patients with diabetes: a comprehensive systematic review and meta-analysis of retrospective studies. Cancer. 2017; 8(13):2532-41.

14. Siwei Z, Huali X, Xiaofeng Y, Wu Y, Sui D. Metformin ameliorates diabetic nephropathy in a rat model of low-dose streptozotocin-induced diabetes. Exp and Therap Med. 2017;14(1):383-90.

15. Hao H, Jung $\mathrm{H}$, Chen L, Chia C, Yueh L, Jang S. Effect of metformin on kidney function in patients with type 2 diabetes mellitus and moderate chronic kidney disease. Oncotarget. 2018; 9(4):5416-23.

16. Le Roux CW, Astrup A, Fujioka K. Obesity Prediabetes NN8022-1839 Study Group. 3 years of liraglutide versus placebo for type 2 diabetes risk reduction and weight management in individuals with prediabetes: a randomized, double-blind trial. Lancet. 2017; 389:1399-409.

17. Matloff DS, Selinger MJ, Kaplan MM. Hepatic transaminase activity in alcoholic liver disease. Gastroenterology. 1980; 78:1389-92.

18. Burstein M, Scholnick HR, Morfin R. Rapid method for the isolation of lipoproteins from human serum by precipitation with polyanions. J. Lipid Res. 1970; 11(6):58-95.

19. Allain CC, Poon LS, Chan CS, Richmond W, Fu PC. Enzymatic determination of total serum cholesterol. Clin Chem. 1974; 20(4):470-5.

20. Fossati $P$, Prencipe L. Serum triglycerides determined calorimetrically with an enzyme that produces hydrogen peroxide. Clin Chem. 1982; 28(10):2077-80.

21. Friedewald WT, Levy RI, Fredrickson DS. Estimation of the concentration of low-density lipoprotein cholesterol in plasma, without use of the preparative ultracentrifuge. Clin Chem. 1972; 18(6):499-502.

22. Paoletti F, Mocali A. Determination of superoxide dismutase activity by purely chemical system based on NAD(P)H oxidation. Methods Enzymol. 1990; 186: 209-20.

23. Aebi H. Catalase in vitro. Methods Enzymol. 1984; 105:121-6.

24. Paglia DE, Valentine WN. Studies on the quantitative and qualitative characterization of erythrocyte glutathione peroxidase. J of Labor and Clin Med. 1967; 70(1):158-69.

25. Singhal A, Jie L, Kumar P, Hong GS, Leow MK, Paleja B, et al. Metformin as adjunct antituberculosis therapy. Sci Trans Med. 2014; 6(263):263ra159.

26. Motta $A B$. Mechanisms involved in metformin action in the treatment of polycystic ovary syndrome. Current Pharmaceut Design. 2009; 15(26):3074-7.

27. Han X, Tao YL, Deng YP, Yu JW, Cai J, Ren GF, et al. Metformin ameliorates insulitis in STZ-induced diabetic mice. Peer J. 2017; 5:e3155.

28. Zhang Z, Li F, Tian Y, Cao L, Gao Q, Zhang C, et al. Metformin Enhances the antitumor activity of CD8+ T Lymphocytes via the AMPK-miR-107-Eomes-PD-1 pathway. Immunology. 2020; 27:1-9.

29. Yanardag R, Sacan O, Bolkent S, Orak H, Karabulut-Bulan O. Protective effects of metformin treatment on the liver injury of streptozotocin-diabetic rats. Human Experim Toxicol. 2005; 24(3):129-35.

30. Li Y, Liu L, Wang B, Wang J, Chen D. Metformin in non- alcoholic fatty liver disease: a systematic review and metaanalysis. Biomed Reports. 2013; 1:57-64.

31. Hassanzadeh-Taheri M, Hassanpour-Fard M, Doostabadi M, Moodi H, Vazifeshenas-Darmiyan K, Hosseini M. Coadministration effects of aqueous extract of turnip leaf and metformin in diabetic rats. J. Trad. Complem. Med. 2018; 8(1):178-83.

32. Rizk FH, Saadany A, Dawood L, Elkaliny HH, Sarhan NI, Badawi R, et al. Metformin ameliorated methotrexateinduced hepatorenal toxicity in rats in addition to its antitumor activity: two birds with one stone. J. Inflam Res. 2018; 11:421-9.

33. Lin SH, Cheng PC, Tu ST, Hsu SR, Cheng YC, Liu YH. Effect of metformin monotherapy on serum lipid profile in statin-naïve individuals with newly diagnosed type 2 diabetes mellitus: a cohort study. Peer J. 2018; 6:e4578. 
34. Patel CR, Patel DA, Patel DJ. Study of effect of metformin on lipid profile in type 2 diabetes mellitus in a tertiary care teaching hospital. Int. J. Pharmaceut. Sci. Res. 2019;10(12):5553-5558.

35. Obi BC, Okoye TC, Okpashi VE, Igwe CN, Alumanah EO. Comparative study of the antioxidant effects of metformin, glibenclamide, and repaglinide in alloxan-induced diabetic rats. Diab Res. 2016; 1635361.

36. Thamer SJ. The effect of orlistat and metformin treatment on body weight, liver steatosis, leptin and insulin sensitivity in obese rats fed high fat diet. American J. Sci. 2014; 10(4):107-14.

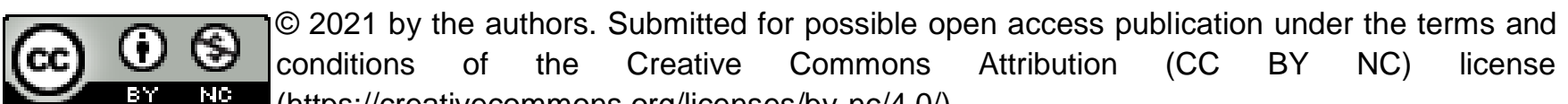
(https://creativecommons.org/licenses/by-nc/4.0/). 